\title{
Dynamic Changes Of Body Image And Quality Of Life In Breast Cancer Patients
}

This article was published in the following Dove Press journal:

Cancer Management and Research

\author{
Tzu-Yi Wu' \\ Tsai-Wang Chang ${ }^{2}$ \\ Sheng-Mao Chang $\mathbb{D}^{3}$ \\ Yun-Ying Lin ${ }^{4}$ \\ Jung-Der Wang (D) $^{5,6}$ \\ Yao-Lung Kuo ${ }^{7}$
}

'Department of Occupational Therapy, College of Medical and Health Science, Asia University, Taichung, Taiwan;

${ }^{2}$ Division of Breast Surgery, Department of Surgery, Tainan Municipal An-Nan Hospital - China Medical University, Tainan, Taiwan; ${ }^{3}$ Department of Statistics, National Cheng Kung University, Tainan, Taiwan; ${ }^{4}$ Department of Medicine, College of Medicine, National Cheng Kung University, Tainan, Taiwan;

${ }^{5}$ Departments of Internal Medicine and Occupational and Environmental Medicine, National Cheng Kung University Hospital, Tainan, Taiwan;

${ }^{6}$ Department of Public Health, College of Medicine, National Cheng Kung University, Tainan, Taiwan; ${ }^{7}$ Department of Surgery, National Cheng-Kung University Hospital, College of Medicine, National Cheng Kung University, Tainan and Dou-Liou, Taiwan
Correspondence: Yao-Lung Kuo Department of Surgery, National ChengKung University Hospital, College of

Medicine, National Cheng Kung

University, No.138, Sheng Li Road, Tainan

704, Taiwan

Tel +886-6-2353535 ext. 5224

Email ylkuo@mail.ncku.edu.tw
Purpose: Dynamic changes of body image and quality of life (QoL) in breast cancer patients were not commonly investigated. We aimed to compare the dynamic changes in QoL and body image of breast cancer survivors receiving breast-conserving surgery or total mastectomy within 5-10 years after surgery.

Methods: Patients with non-metastatic breast cancer who received surgery were invited to complete the World Health Organization Quality of Life-Brief (WHOQOL-BREF) questionnaire and the Body Image Scale (BIS) within 10 years after surgery. We applied kernel smoothing methods to capture the dynamic changes of the patients' QoL and body image within 5 years after surgery. We also constructed multiple linear regression models to identify predictive factors for QoL and body image.

Results: A total of 581 patients were collected, and 211 of them received breast-conserving surgery. There were no statistically significant differences in QoL and body image for breastconserving surgery versus total mastectomy, but the former showed fluctuating trends. BIS was a predictor of every item and domain in the WHOQOL-BREF in the multiple linear regression model, and explanatory of the trends of dynamic change over time. Patients without lymph node dissection seemed to have less positive feelings but were more satisfied with sexual activities.

Conclusion: Body image is predictive of the QoL of breast cancer patients. Dynamic changes of body image and QoL would be useful for shared decision-making regarding surgery in breast cancer patients.

Keywords: breast cancer survivors, body image, breast-conserving surgery, quality of life, total mastectomy

\section{Introduction}

Breast cancer is one of the most common cancer types in women worldwide. ${ }^{1,2}$ It ranks first among Taiwanese women ${ }^{3}$ and is reported as the fourth leading cause of death from cancer in Taiwan. ${ }^{4}$ According to worldwide statistics, the five-year survival rate of patients with breast cancer in early stage without metastasis is $89 \%{ }^{5}$

Because the life expectancy of breast cancer is among the longest in cancer, or, approximately 23 years, ${ }^{3}$ quality of life (QoL) in survivors is a major concern and should be considered beginning from the first clinical encounter and management. ${ }^{6}$ In addition, body image in patients with breast cancer differs from all other cancers, because the sequelae from treatment, including surgery, chemotherapy, hormone therapy, radiotherapy or the above interventions combined, may directly interfere with daily life and social functions. ${ }^{7}$ Among them, surgical interventions of the 
breast have the most impact on women psychologically and socially. ${ }^{8}$ Therefore, decisions related to different surgical modalities, such as conservation of breast and dissection of axillary lymph nodes, are necessary in treating such patients. ${ }^{9}$

Studies regarding QoL and body image in Asian patients with breast cancer are relatively lacking and usually conducted with short-term follow-up. ${ }^{10}$ To evaluate the impacts of breast cancer treatment on patients' QoL and body image, we chose two widely used questionnaires, the World Health Organization Quality of Life-Brief Questionnaire (WHOQOL-BREF) and the Body Image Scale (BIS) for assessment. This study aimed to compare the long-term quality of life and body image of breast cancer survivors receiving breastconserving surgery or total mastectomy and axillary lymph node dissection or sentinel lymph node biopsy within 10 years after surgery. By applying the kernel smoothing method, we also explored the dynamic changes of breast cancer survivors' quality of life and body image within 5 years after surgery.

\section{Materials And Methods}

\section{Participants}

The study was approved by the Institutional Review Board of National Cheng Kung University Hospital before commencement (A-ER-105-191). Patients with pathologically verified breast cancer were abstracted from the cancer registry of the Oncology Center of National Cheng Kung University Hospital. The database also stores the records of self-reported questionnaires of cancer patients who visited National Cheng Kung University Hospital with informed consent. All identification numbers were encrypted in the final data analysis. The inclusion criteria for the participants were as follows: (1) aged at least 18 years old; (2) breast cancer with ICD-O-3 (International Classification of Diseases - Oncology, 3rd edition) C50.0-50.9 and a pathological stage of $0-3$, according to the American Joint Committee on Cancer Staging Manual (7th edition); (3) receiving breast-conserving surgery, total mastectomy or modified radical mastectomy; (4) still within 10 years after surgery. Subjects who were unable to read or answer the questionnaires, had a recurrent event, or had bilateral breast cancer were excluded. In addition, we excluded patients receiving breast reconstruction because only limited number of patients received this surgery in the Oncology Center of National Chang Kung
University Hospital. Their QoL would be analyzed after collection of a sufficient sample size.

\section{Procedures}

All eligible candidates who came to Oncology Center of National Cheng Kung University Hospital for regular follow-up were invited to participate in the study. The participants were instructed to self-complete the Taiwan version of the WHOQOL-BREF questionnaire and the BIS via tablet computers. An experienced research assistant was available to clarify the meaning of the items in a standardized way if any questions were raised. The patients' demographic information and clinical data were abstracted from electronic medical records and the cancer registry of National Cheng Kung University Hospital.

\section{Measurements Of QoL And Body Image}

The World Health Organization Quality-of-Life-Brief (WHOQOL-BREF) is a generic QoL questionnaire, which was developed under WHO supervision ${ }^{11}$ and provided in the Supplementary material. The Taiwan version of the WHOQOL-BREF is composed of 28 items, comprising physical, psychological, social and environment domains and two local items ("being respected" and "eating"). ${ }^{12}$ All items are rated on a 5-point Likert scale. A higher score indicates better quality of life. The Taiwan version of the WHOQOL-BREF has been validated to have good internal consistency reliability (Cronbach's $\alpha>0.91$ ), testretest reliability (correlation coefficient $>0.75$ ) and construct validity in patients with malignancies. ${ }^{12-14}$

The BIS is a valid measure for body image discomfort in patients with cancer, ${ }^{15,16}$ and the items of the BIS can be found in reference 16. The BIS has 10 items enclosing three concepts: affective, behavioral, and cognitive body image symptoms. Each item is rated on 4-level scale: 0 (not at all), 1 (a little), 2 (quite a bit), and 3 (very much). The score of each item can be summed as a total score ranging from 0 to 30. A higher total score indicates increasing symptoms/ distress of changes in cancer-related body image. The internal consistency of the BIS was good (Cronbach's $\alpha=0.90$ ) in Taiwanese patients with breast cancer. ${ }^{15}$

The Barthel Index is a measurement to evaluate people's functional independence in basic activities of daily living. ${ }^{17}$ It contains 10 items: feeding, bathing, grooming, dressing, bowel control, bladder control, toilet use, transfers (bed to chair and back), mobility on level surfaces, and stair climbing. The sum score of the Barthel Index ranges from 0 to $100 .{ }^{18} \mathrm{~A}$ higher sum score indicates that 
the patient has better functional independence. The Barthel Index has been well validated to have high internal consistency (Cronbach's $\alpha=0.82$ ), high concurrent validity (Spearman correlation coefficient $=0.92$ ), and high responsiveness (standardized response mean $=1.2) .{ }^{19}$

\section{Statistical Analysis}

A kernel-type smoother was used to illustrate the dynamic changes in item scores, ${ }^{20}$ domain scores, and/or total scores of the Taiwan version of WHOQOL-BREF and BIS using an open access software, the $\mathrm{R}$ software. Patients without recurrence and reconstruction were grouped into the breastconserving surgery group and total mastectomy group. We compared the trends in QoL changes of sentinel lymph node biopsy and axillary lymph node dissection patients within the breast-conserving surgery and total mastectomy groups. Because the number of participants decreased with time, the timeline of dynamic changes was depicted within 5 years after breast cancer surgery.

Multiple linear regression models were constructed to determine the associations between the scores of each item and domain of the WHOQOL-BREF/body image/activity of daily living and methods of surgery (ie, breast-conserving surgery and total mastectomy) after controlling potential confounding factors, including age, hormone therapy, marital status, education level, and family income (more or less than USD \$1650/month), co-morbidities, pathological stage, types of lymph node dissection, time after chemotherapy ( $\geq 18$ vs $<18$ months), time after radiotherapy ( $\geq 12$ vs $<12$ months), time after surgery, and interaction terms of surgery and lymph node dissection. The above potential confounding factors were included into the multiple linear regressions based on literature review about the QoL of patients with breast cancer. $^{21,22}$ We have also checked and controlled for collinearity. ${ }^{23}$ In addition, models were also constructed with the total scores of BIS and dependence in activity of daily living (ie, the score of Barthel index $<100$ ) as independent variables to test the hypotheses that both scores would affect many items and domains of QoL. Multiple linear regression models were also performed using the $\mathrm{R}$ software.

\section{Results}

\section{Demographics And Clinical Characteristics Of The Participants}

A total of 581 breast cancer patients with pathologic stages of 0 to 3 completed the WHOQOL-BREF and the BIS. Table 1 compares the demographic and clinical characteristics among participants receiving breastconserving surgery and total mastectomy. One-third $(36.3 \%)$ of them received breast-conserving surgery. On average, patients receiving total mastectomy were diagnosed

Table I Demographic And Clinical Characteristics Of Breast Cancer Patients. ( $\mathrm{N}=58 \mathrm{I})$

\begin{tabular}{|c|c|c|c|}
\hline & $\begin{array}{l}\text { Breast- } \\
\text { Conserving } \\
\text { Surgery }\end{array}$ & $\begin{array}{l}\text { Total } \\
\text { Mastectomy }\end{array}$ & $p$ \\
\hline Total no. of patients & 211 & 370 & \\
\hline $\begin{array}{l}\text { Age (year); Mean } \pm S D \\
\quad \geq 55 \text { y/o }\end{array}$ & $\begin{array}{l}52.3 \pm 8.8 \\
79(37.4 \%)\end{array}$ & $\begin{array}{l}53.7 \pm 9.2 \\
154(41.6 \%)\end{array}$ & $\begin{array}{l}0.06 \\
0.32\end{array}$ \\
\hline $\begin{array}{l}\text { Education } \leq 6 \text { years } \\
7-12 \text { years } \\
>12 \text { years }\end{array}$ & $\begin{array}{l}29(13.7 \%) \\
93(44.1 \%) \\
86(40.8 \%)\end{array}$ & $\begin{array}{l}56(15.1 \%) \\
178(48.1 \%) \\
135(36.5 \%)\end{array}$ & 0.93 \\
\hline Married or cohabited & 156 (73.9\%) & $268(72.4 \%)$ & 0.70 \\
\hline Income $^{\mathrm{a}}>$ US $\$ 1646 /$ month & $129(61.1 \%)$ & 207 (56.9\%) & 0.32 \\
\hline $\begin{array}{l}\text { Pathologic stage } 0 \\
\text { I } \\
\text { II } \\
\text { III }\end{array}$ & $\begin{array}{l}25(11.8 \%) \\
119(56.4 \%) \\
59(28.0 \%) \\
8(3.8 \%)\end{array}$ & $\begin{array}{l}46(12.4 \%) \\
97(26.2 \%) \\
163(44.1 \%) \\
64(17.3 \%)\end{array}$ & $<0.001$ \\
\hline Lymph node dissection (-) & II (5.2\%) & II (2.9\%) & $<0.001$ \\
\hline $\begin{array}{l}\text { Sentinel lymph node } \\
\text { biopsy }\end{array}$ & 142 (67.3\%) & $126(34.1 \%)$ & \\
\hline $\begin{array}{l}\text { Axillary lymph node } \\
\text { dissection }\end{array}$ & $58(27.5 \%)$ & $233(63.0 \%)$ & \\
\hline $\begin{array}{l}\text { Months after surgery } \\
\text { (Mean } \pm \text { SD) } \\
\text { Within I year } \\
\text { I-5 years } \\
>5 \text { years }\end{array}$ & $\begin{array}{l}33.3 \pm 30.9 \\
75(35.5 \%) \\
96(45.5 \%) \\
40(19.0 \%)\end{array}$ & $\begin{array}{l}32.2 \pm 32.0 \\
147(39.7 \%) \\
142(38.4 \%) \\
8 \mid(21.9 \%)\end{array}$ & $\begin{array}{l}0.66 \\
0.24\end{array}$ \\
\hline $\begin{array}{l}\text { Chemotherapy: Never } \\
\leq 6 \text { months } \\
>6 \text { months }\end{array}$ & $\begin{array}{l}90(42.7 \%) \\
20(9.5 \%) \\
101(47.8 \%)\end{array}$ & $\begin{array}{l}125(33.8 \%) \\
51(13.8 \%) \\
194(52.4 \%)\end{array}$ & 0.07 \\
\hline $\begin{array}{l}\text { Radiotherapy: Never } \\
\leq 2 \text { years } \\
>2 \text { years }\end{array}$ & $\begin{array}{l}51(24.2 \%) \\
72(34.1 \%) \\
88(41.7 \%)\end{array}$ & $\begin{array}{l}263(71.1 \%) \\
47(12.7 \%) \\
60(16.2 \%)\end{array}$ & $<0.001$ \\
\hline Hormone therapy $<10$ years & $143(67.8 \%)$ & $215(58.1 \%)$ & 0.02 \\
\hline Diabetes mellitus (yes) & $13(6.2 \%)$ & $33(8.9 \%)$ & 0.22 \\
\hline Heart Disease (yes) & $6(2.8 \%)$ & $9(2.4 \%)$ & 0.76 \\
\hline $\begin{array}{l}\text { Total independence in } \\
\text { activity of daily living }\end{array}$ & 187 (95.4\%) & 319 (91.1\%) & 0.18 \\
\hline Body image scale (10-36) & $13.6 \pm 4.9$ & $14.8 \pm 5.3$ & 0.01 \\
\hline
\end{tabular}

Note: a Family income. 
at more advanced pathological stages, which were also accompanied with higher proportions receiving axillary lymph node dissection, lower proportions receiving radiotherapy and hormone therapy, compared with breastconserving surgery groups. The other characteristics showed no significant differences between the two groups of participants.

\section{Dynamic Changes Of QoL And Body Image}

Compared with patients receiving total mastectomy, scores of QoL and BIS in patients receiving breast-conserving surgery tended to show fluctuations with time after surgery, especially in patients receiving axillary lymph node dissection (Figures 1 and 2). On closer examination, patients receiving breast-conserving surgery and axillary lymph node dissection seemed to show decreasing trends in the scores of "medication", "sleep and rest", "work capacity", "negative feeling", and "being respected". They also tended to feel "dissatisfied with appearance" and "less sexually attractive" at about 40 months after surgery. In contrast, patients receiving total mastectomy showed relatively stable trends for groups with sentinel lymph node biopsy or axillary lymph node dissection. Patients receiving axillary lymph node dissection seemed to feel "less physically attractive" in the BIS over the first 5 years. On the item of "body image" in the WHOQOLBREF, patients receiving axillary lymph node dissection seemed to show an improving trend within 5 years after

\section{Breast Conservation Surgery Total Mastectomy}

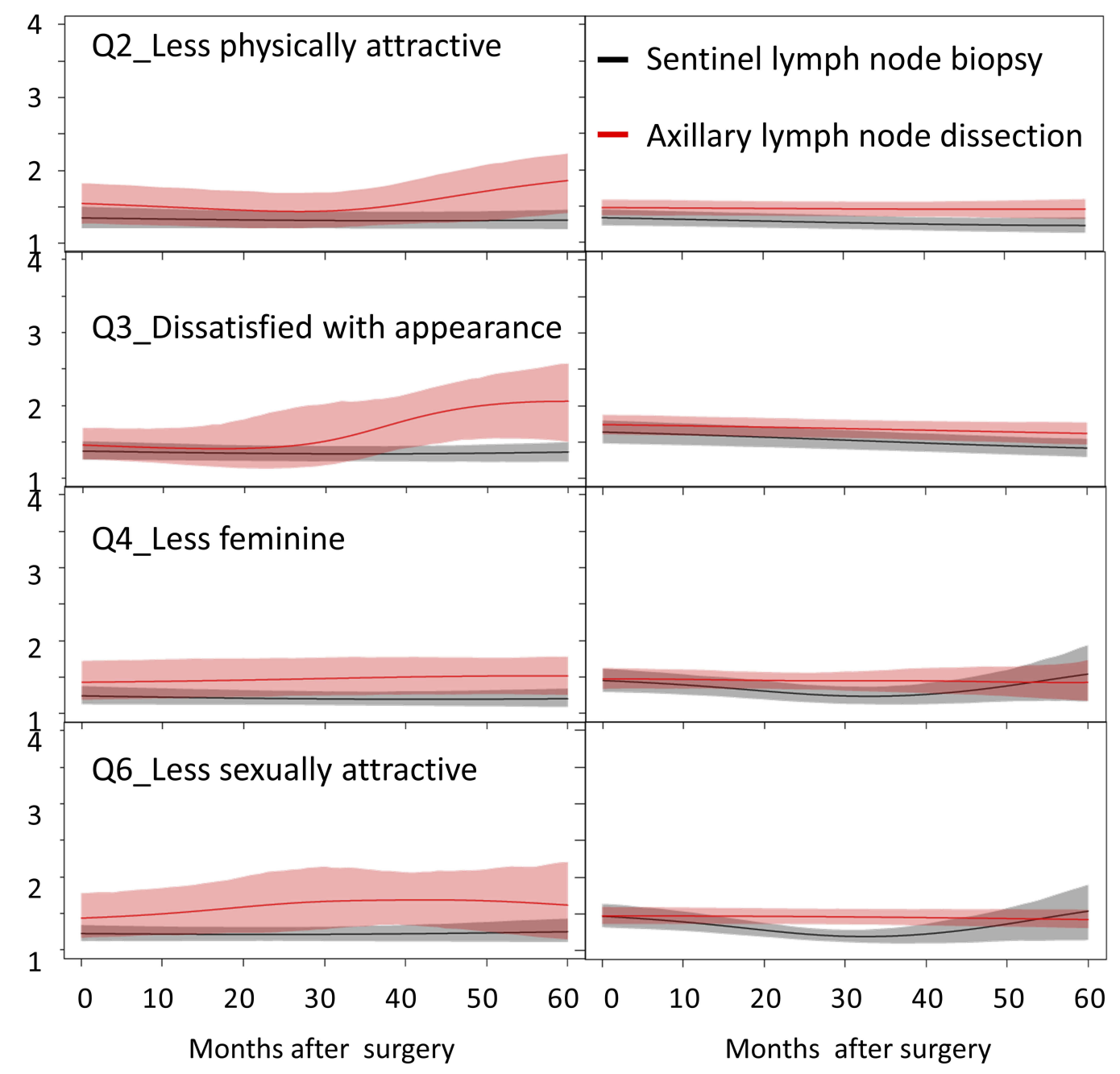

$\begin{array}{lccccccccccccc}\text { Sentinel lymph node biopsy } & 43 & 22 & 15 & 14 & 19 & 7 & 67 & 12 & 15 & 15 & 5 & 5 \\ \text { Axillary lymph node dissection } & 22 & 7 & 3 & 1 & 4 & 6 & 71 & 26 & 18 & 17 & 18 & 12\end{array}$

Figure I Dynamic changes of scores of the Body Image Scale in patients with breast cancer stratified by types of surgery and lymph node dissection. 


\section{Breast Conservation Surgery Total Mastectomy}

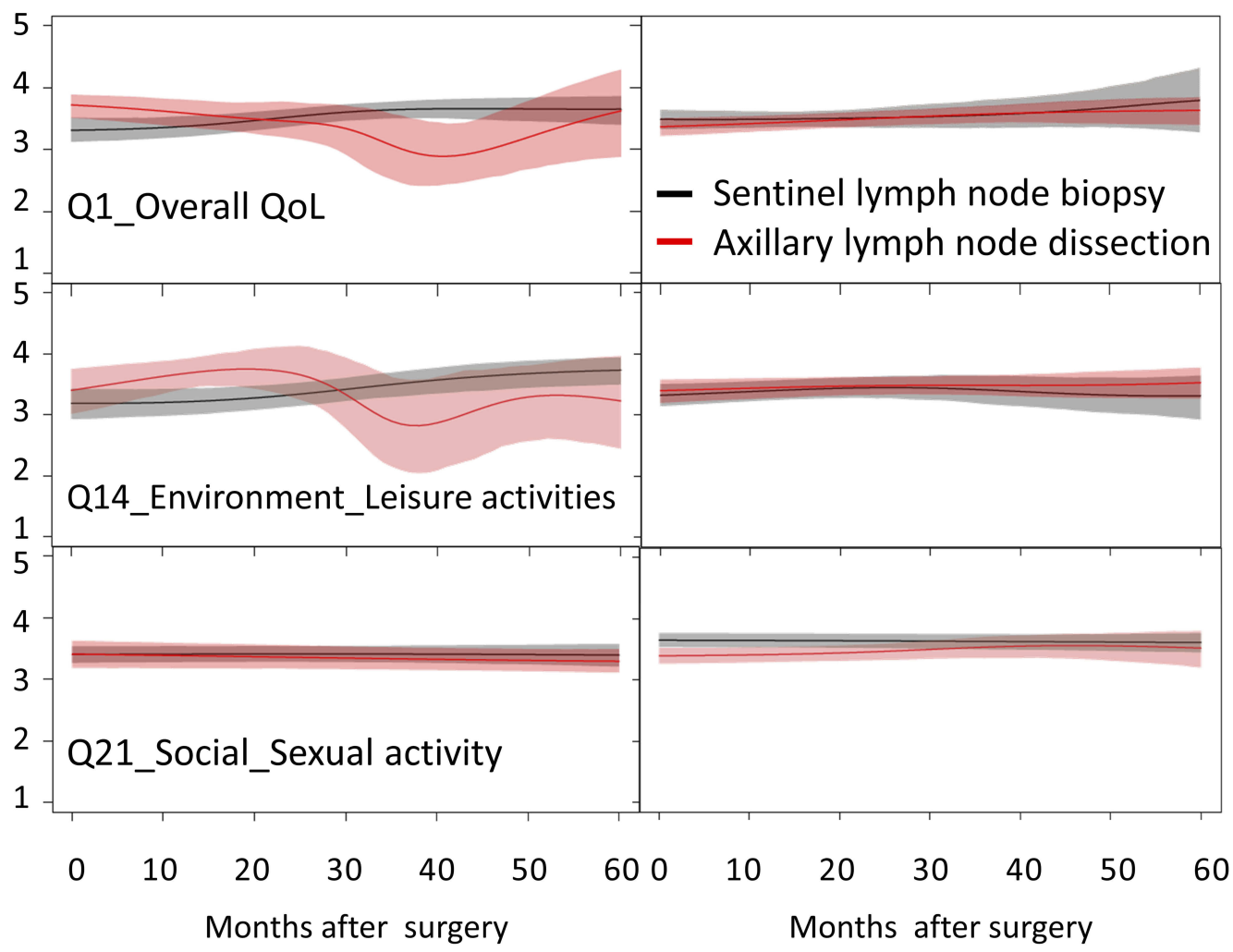

$\begin{array}{lcccccccccccc}\text { Sentinel lymph node biopsy } & 43 & 22 & 15 & 14 & 19 & 7 & 67 & 12 & 15 & 15 & 5 & 5 \\ \text { Axillary lymph node dissection } & 22 & 7 & 3 & 1 & 4 & 6 & 71 & 26 & 18 & 17 & 18 & 12\end{array}$

Figure 2 Dynamic changes of scores of the WHOQOL-BREF in patients with breast cancer according to the surgery type.

surgery, whereas patients receiving sentinel lymph node biopsy showed a fluctuating trend. No significant differences in trends were found between those receiving sentinel lymph node biopsy and axillary lymph node dissection.

\section{Multiple Linear Regression For Exploring Determinants Of QoL And Body Image}

No collinearity existed in the models (all variance inflation factors $<10$ ). Table 2 summarizes the results of the multiple linear regression models. We found that the total score of the BIS was associated with every item and domain of the WHOQOL-BREF while that of activity of daily living was associated with most items in the physical, psychological, and environment domains. Age, family income, and $<6$ months after chemotherapy were predictive of scores of some items in the QoL and BIS.

After controlling for potential confounders, we found that patients' QoL was not associated with different types of tumor removing surgery. Patients without lymph node dissection seemed to have less positive feelings but were more satisfied with sexual activities, compared to patients receiving axillary lymph node dissection. Patients receiving sentinel lymph node biopsy less frequently felt physically unattractive due to their illness, compared to patients receiving axillary lymph node dissection. There appeared to be some interaction between the types of tumor removal surgery and lymph node dissection: Patients receiving breast-conserving surgery and without lymph node dissection had higher scores on the item of "safety and security", and lower scores on the item of "leisure activities".

\section{Discussion}

After stratifying the patients according to different types of breast and axillary lymph node surgery, we found that patients receiving total mastectomy without reconstruction showed relatively stable trends, compared with patients receiving breast-conserving surgery and patients receiving 
Table 2 Regression Coefficients Of Each Domain And Item Scores Of The WHOQOL-BREF Based On Multiple Linear Regression Models; Those With No Statistical Significance ( $P>0.05)$ Were Not Listed

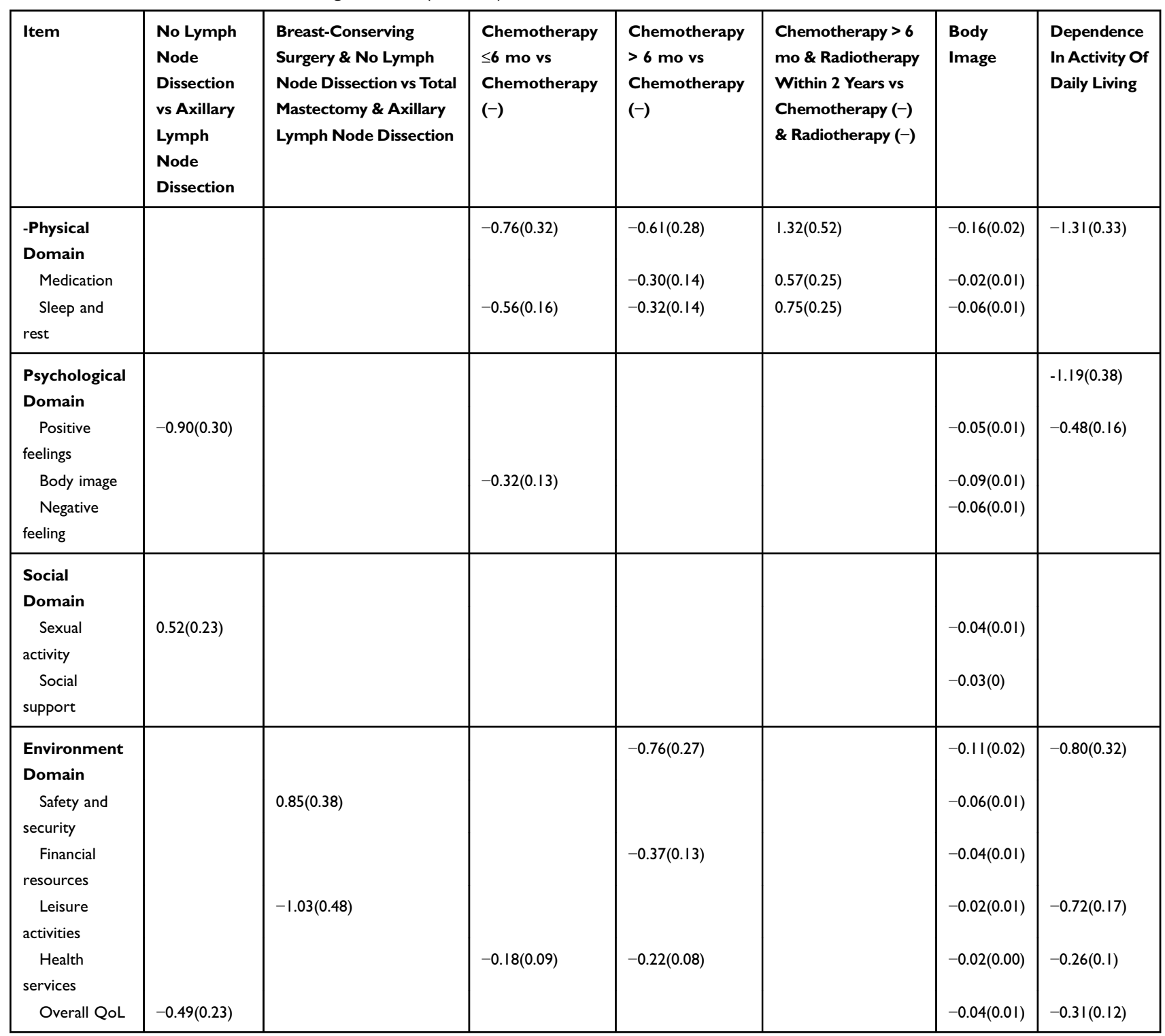

Notes: All models have controlled following confounders: age, age groups ( $>55 /<55$ years old), hormone therapy (yes/no), marital status (yes/no), 3 education levels, and family income ( $>/<$ USD $\$ 1650 /$ month), co-morbidities, pathological stage, types of lymph node dissection (sentinel lymph node biopsy/(-)), and axillary lymph node dissection/(-)), time after first chemotherapy ( $>18 /<18$ months), time after first radiotherapy $(>13 /<13$ months), time after surgery $(<1 / 1-5 />5$ years), and interactions of types of surgery and lymph node dissection.

Abbreviations: Chemotherapy (-), never chemotherapy; Chemotherapy $\leq 6$ mo, within 6 months after the first chemotherapy; Chemotherapy $>6$ mo, beyond 6 months after the first chemotherapy; QoL, quality of life; Radiotherapy (-), never radiotherapy; Dependence in Dependence of activity of daily living, the score of the Barthel Index $<100$.

axillary lymph node dissection, who appeared to show wider fluctuations in scores of QoL and body image with time after surgery. The results of multiple linear regression showed that educational level and time after surgery were common factors associated with scores of patients' QoL and body image. The total score of the BIS is predictive of the scores of all 28 items and 4 domains of the WHOQOLBREF (Taiwan version) after controlling other predictive factors, while that of the Barthel index is independently predictive of 11 items and 3 domains (Table 2). Moreover, the types of surgery did not show any significant differences in the scores of QoL after controlling BIS and Barthel index. Lymph node dissection significantly diminished the scores of "positive feeling" and overall health of WHOQOL-BREF, while it improved the score of sexual life. The influence of surgical methods can be explained by changes in body image and functions. Patients of breast cancer who did not receive lymph node dissection 
appeared to continually worry about recurrence. These findings would be useful to breast cancer patients for choosing surgical methods and shared decision-making.

Compared with other patients, the scores of QoL and body image in patients receiving breast-conserving surgery and axillary lymph node dissection fluctuated at larger variances with time after surgery (Figures 1 and 2). Before any operation, it is difficult to imagine the extent of the scar and postoperative appearance of the breast after receiving breast-conserving surgery and postoperative irradiation. Patients with breast-conserving surgery would have better expectations of body image, which frequently results in decreased satisfaction and fluctuations of subjective QoL 2 years after surgery (Figures 1 and 2). Moreover, patients receiving axillary lymph node dissection were more likely to have a long-term lymph edema after breast surgery, ${ }^{24}$ which would affect patients' appearance of body image and QoL. Whereas, patients receiving total mastectomy were usually informed that there would be a long permanent scar on the chest, but they might be less worried and expect a lower probability of recurrence after removing the entire mammary gland. Accordingly, patients receiving breast-conserving surgery may have worse, fluctuating scores of QoL and body image, because they are frequently unsatisfied with the shape of their breast and are constantly worried about recurrence. Besides, the differences between sentinel lymph node biopsy and axillary lymph node dissection in patients receiving total mastectomy were not as obvious as those receiving breast-conserving surgery in our study, because the former usually have lower expectations about the postoperative appearance of their breasts. Our results on the long-term dynamic trends of QoL and body image would be useful for clinicians to provide timely interventions at important turning points.

After controlling potential confounding factors, our study showed that types of breast surgery make no significant differences on patients' QoL and body image. Instead, these outcomes were affected by chemotherapy or radiotherapy or being comorbid with heart disease. Our results appear to corroborate with other Asian studies on major predictive factors influencing the QoL of patients with breast cancer. ${ }^{10,25}$ However, there seems to be no consensus on whether breast-conserving surgery or total mastectomy is better for patients' QoL and body image. ${ }^{26-28}$ Culture and surgeon's skill may also affect patients' preferences on the types of surgery and expectations of treatment outcomes, leading to results with wide variations. More research with long-term repeated measurements is needed to determine whether breast-conserving surgery is indeed better than total mastectomy.

Our study has at least two strengths. First, we illustrated the dynamic changes of patients' QoL and body image for 5 years after the surgery based on real-world data, which would be useful for patients to gain a more realistic expectation of the prognosis. Second, the construction of our statistical model corroborates the common notion that subjective feelings of one's body image significantly affect every facet and domain of QoL, including socio-environmental ones (Table 2). The phenomenon seems more easily demonstrated in patients with breast cancer. However, this study also has the following limitations. First, the participants in this study were recruited from a single tertiary medical center in Southern Taiwan. The results must be tested in different settings, regions, and/or cultures, before any generalization can be made. Second, this study excluded patients with bilateral or recurrent breast cancer. Future studies are needed for those with the above characteristics. Third, we were unable to consider the level of surgeons' operational skills, patients' body mass index and bra cup size in our model construction. However, these factors would generally influence the QoL through change of BIS scores and would usually not confound the effects of various predictors listed in Table 2. Future studies are warranted to take these factors into account, especially for exploration of determinants of BIS.

\section{Conclusions}

By using kernel smoothing methods in this pilot study, we found that patients receiving total mastectomy without reconstruction showed relatively stable trends, compared with patients receiving breast-conserving surgery; patients receiving axillary lymph node dissection tended to show fluctuations in scores of QoL and body image with time after surgery. The total scores of body image are predictive of all facets and domains of QoL, and the Barthel index is predictive of many facets and 3 domains, implying the relevance of functional states after treatment. The dynamic changes of scores of QoL and body image in patients with breast cancer would be useful for shared clinical decision-making and informing healthcare workers about when and how to help improve the QoL of cancer survivors. 


\section{Abbreviations}

BIS, Body Image Scale; QoL, quality of life; WHOQOLBREF, World Health Organization Quality of Life-Brief.

\section{Acknowledgments}

This study was partially supported by research grants from the Ministry of Science and Technology (MOST105-2314B-006-032), Establishment of Cancer Research System Excellence Program funded by the Ministry of Health and Welfare, Taiwan (MOHW103-TD-B-111-06). The funders had no role in the study design, data collection and analysis, decision to publish, or preparation of the manuscript. We are grateful to the Biostatistics Consulting Center, National Cheng Kung University Hospital for providing the statistical consulting services.

\section{Disclosure}

The authors report no conflicts of interest in this work.

\section{References}

1. Siegel RL, Miller KD, Jemal A. Cancer statistics, 2017. CA Cancer J Clin. 2017;67(1):7-30. doi:10.3322/caac.21387

2. Torre LA, Siegel RL, Ward EM, Jemal A. Global cancer incidence and mortality rates and trends-an update. Cancer Epidemiol Biomarkers Prev. 2016;25(1):16-27. doi:10.1158/1055-9965.EPI-150578

3. Wu TY, Chung CH, Lin CN, Hwang JS, Wang JD. Lifetime risks, loss of life expectancy, and health care expenditures for 19 types of cancer in Taiwan. Clin Epidemiol. 2018;10:581-591. doi:10.2147/ CLEP.S155601

4. Ministry of Health and Welfare. Taiwan cancer registry annual report in 2015. Taiwan: Health Promotion Administration; 2017.

5. Miller KD, Siegel RL, Lin CC, et al. Cancer treatment and survivorship statistics, 2016. CA Cancer J Clin. 2016;66(4):271-289. doi:10. 3322/caac.v66.4

6. Van Leeuwen M, Husson $\mathrm{O}$, Alberti $\mathrm{P}$, et al. Understanding the quality of life (QOL) issues in survivors of cancer: towards the development of an EORTC QOL cancer survivorship questionnaire. Health Qual Life Outcomes. 2018;16(1):114. doi:10.1186/s12955018-0920-0

7. Paterson CL, Lengacher CA, Donovan KA, Kip KE, Tofthagen CS. Body image in younger breast cancer survivors: a systematic review. Cancer Nurs. 2016;39(1):E39-58. doi:10.1097/NCC.0000000000000 251

8. Campbell-Enns H, Woodgate R. The psychosocial experiences of women with breast cancer across the lifespan: a systematic review protocol. JBI Database Syst Rev Implementation Rep. 2015;13 (1):112-121. doi:10.11124/jbisrir-2015-1795

9. Durand MA, Yen RW, O'Malley AJ, et al. What matters most: protocol for a randomized controlled trial of breast cancer surgery encounter decision aids across socioeconomic strata. BMC Public Health. 2018;18(1):241. doi:10.1186/s12889-018-5109-2

10. Ho PJ, Gernaat SAM, Hartman M, Verkooijen HM. Health-related quality of life in Asian patients with breast cancer: a systematic review. BMJ Open. 2018;8(4):e020512. doi:10.1136/bmjopen-2017020512
11. Harper A. Development of the World Health Organisation WHOQOL-BREF quality of life assessment. Psychol Med. 1998;28 (3):551-558. doi:10.1017/S0033291798006667

12. Yao G, Chung CW, Yu CF, Wang JD. Development and verification of validity and reliability of the WHOQOL-BREF Taiwan version. $J$ Formos Med Assoc. 2002;101(5):342-351.

13. Yao G. Development of the WHOQOL-BREF Taiwan Version and the Manual. The WHOQOL-Taiwan Group; 2005.

14. Lin CY, Hwang JS, Wang WC, et al. Psychometric evaluation of the WHOQOL-BREF, Taiwan version, across five kinds of Taiwanese cancer survivors: Rasch analysis and confirmatory factor analysis. J Formos Med Assoc. 2018;118:215-222.

15. Fang SY, Chang HT, Shu BC. Objectified body consciousness, body image discomfort, and depressive symptoms among breast cancer survivors in Taiwan. Psychol Women Q. 2014;38(4):563-574. doi:10. 1177/0361684314552652

16. Hopwood P, Fletcher I, Lee A, Al Ghazal S. A body image scale for use with cancer patients. Eur J Cancer. 2001;37(2):189-197. doi:10. 1016/S0959-8049(00)00353-1

17. Mahoney FI, Barthel DW. Functional evaluation: the Barthel Index: a simple index of independence useful in scoring improvement in the rehabilitation of the chronically ill. Md State Med J. 1965.

18. Collin C, Wade D, Davies S, Horne V. The Barthel ADL Index: a reliability study. Int Disabil Stud. 1988;10(2):61-63. doi:10.3109/ 09638288809164103

19. Hsueh IP, Lin JH, Jeng JS, Hsieh CL. Comparison of the psychometric characteristics of the functional independence measure, 5 item Barthel index, and 10 item Barthel index in patients with stroke. J Neurol Neurosurg Psychiatry. 2002;73(2):188-190. doi:10.1136/ jnnp.73.2.188

20. Hwang JS, Wang JD. Integrating health profile with survival for quality of life assessment. Qual Life Res. 2004;13(1):1-10. doi:10. 1023/B:QURE.0000015299.45623.38

21. Tsai HY, Kuo RN, Chung KP. Quality of life of breast cancer survivors following breast-conserving therapy versus mastectomy: a multicenter study in Taiwan. Jpn J Clin Oncol. 2017;47(10): 909-918. doi:10.1093/jjco/hyx099

22. Lavdaniti M, Owens DA, Liamopoulou P, et al. Factors influencing quality of life in breast cancer patients six months after the completion of chemotherapy. Diseases (Basel, Switzerland). 2019;7(1):26.

23. Kutner MH. Applied Linear Statistical Models. Boston: McGraw-Hill Irwin; 2005.

24. Taghian NR, Miller CL, Jammallo LS, O'Toole J, Skolny MN. Lymphedema following breast cancer treatment and impact on quality of life: a review. Crit Rev Oncol Hematol. 2014;92(3):227-234. doi:10.1016/j.critrevonc.2014.06.004

25. Frost MH, Hoskin TL, Hartmann LC, Degnim AC, Johnson JL, Boughey JC. Contralateral prophylactic mastectomy: long-term consistency of satisfaction and adverse effects and the significance of informed decision-making, quality of life, and personality traits. Ann Surg Oncol. 2011;18(11):3110-3116. doi:10.1245/s10434-0111917-7

26. Huang CC, Lien HH, Tu SH, et al. Quality of life in Taiwanese breast cancer survivors with breast-conserving therapy. J Formos Med Assoc. 2010;109(7):493-502. doi:10.1016/S0929-6646(10)60083-6

27. Kim MK, Kim T, Moon HG, et al. Effect of cosmetic outcome on quality of life after breast cancer surgery. Eur J Surg Oncol. 2015;41 (3):426-432. doi:10.1016/j.ejso.2014.12.002

28. van der Steeg AFW, De Vries J, Roukema JA. The value of quality of life and health status measurements in the evaluation of the well-being of breast cancer survivors. Eur J Surg Oncol. 2008;34 (11):1225-1230. doi:10.1016/j.ejso.2007.10.009 


\section{Publish your work in this journal}

Cancer Management and Research is an international, peer-reviewed open access journal focusing on cancer research and the optimal use of preventative and integrated treatment interventions to achieve improved outcomes, enhanced survival and quality of life for the cancer patient.
The manuscript management system is completely online and includes a very quick and fair peer-review system, which is all easy to use. Visit http://www.dovepress.com/testimonials.php to read real quotes from published authors.

Submit your manuscript here: https://www.dovepress.com/cancer-management-and-research-journal 